Pacific Journal of Mathematics

TORSION ENDOMORPHIC IMAGES OF MIXED ABELIAN 


\title{
TORSION ENDOMORPHIC IMAGES OF MIXED ABELIAN GROUPS
}

\author{
ElBert A. WALKer
}

In this paper we will answer Fuchs' PROBLEM 32 (a), and the corresponding part of his PROBLEM 33. (See [1], pg. 203.) The statements of these PROBLEMS are the following.

I. "Which are the torsion groups $T$ that are endomorphic images of all groups containing them as maximal torsion subgroups?"

II. "Which are the torsion groups $T$ such that a basic subgroup of $T$ is an endomorphic image of any group $G$ containing $T$ as its maximal torsion subgroup?"

Actually, we will answer question II and the following question which is more general than I.

III. What groups $H$ are endomorphic images of all groups $G$ containing $H$ such that $G / H$ is torsion free?

The solutions will be effected by using some homological results of Harrison [2]. All groups considered here will be Abelian. The definitions and results stated in the remainder of this paragraph are due to Harrison, and may be found in [2]. A reduced group $G$ is cotorsion if $\operatorname{Ext}(A, G)=0$ for all torsion free groups $A$. If $H$ is a reduced group, then Ext $(Q \mid Z, H)=H^{\prime}$ is cotorsion, where $Q$ and $Z$ denote the additive group of rationals and integers, respectively. Furthermore, $H$ is a subgroup of $H^{\prime}$, (that is, there is a natural isomorphism of $H$ into $H^{\prime}$ ) and $H^{\prime} / H$ is divisible torsion free. This implies, of course, that if $T$ is a torsion reduced group, then $T$ is the torsion subgroup of $T^{\prime}=\operatorname{Ext}(Q / Z, T)$.

Now it is easy to see that if $G$ is a group such that $\operatorname{Ext}(A, G)=0$ for all torsion free groups $A$, then any homomorphic image of $G$ is the direct sum of a cotorsion group and a divisible group. In fact, let $H$ be a homomorphic image of $G$. This gives us an exact sequence

$$
0 \rightarrow K \rightarrow G \rightarrow H \rightarrow 0
$$

which yields the exact sequence

$$
\begin{aligned}
0 \rightarrow \operatorname{Hom}(A, K) & \rightarrow \operatorname{Hom}(A, G) \rightarrow \operatorname{Hom}(A, H) \rightarrow \\
\operatorname{Ext}(A, K) & \rightarrow \operatorname{Ext}(A, G) \rightarrow \operatorname{Ext}(A, H) \rightarrow 0 .
\end{aligned}
$$

If $A$ is any torsion free group, then $\operatorname{Ext}(A, G)=0$, and so $\operatorname{Ext}(A, H)=0$. Write $H=D \oplus L$, where $D$ is the divisible part of $H$. Then $L$ is reduced, and $0=\operatorname{Ext}(A, D \oplus L) \cong \operatorname{Ext}(A, D) \oplus \operatorname{Ext}(A, L)=\operatorname{Ext}(A, L)$, so that $L$ is cotorsion. Our assertion is proved.

Received April 28, 1960. 
Now we are ready to give the solutions promised earlier. The following theorem settles III.

THeOREM. The group $H$ is an endomorphic image of every group $G$ containing it such that $G / H$ is torsion free if and only if $H=D \oplus C$, where $D$ is divisible and $C$ is cotorsion. This is equivalent to the assertion that $H$ is a direct summand of every such $G$.

Proof. Suppose $H$ is an endomorphic image of every group $G$ containing it such that $G / H$ is torsion free. Let $H=D \oplus C$, where $D$ is divisible and $C$ is reduced. Then $C$ is a subgroup of the cotorsion group $\operatorname{Ext}(Q / Z, C)=C^{\prime}$ such that $C^{\prime} / C$ is torsion free, so that $H$ is a subgroup of $D \oplus C^{\prime}=H^{\prime}$ such that $H^{\prime} / H$ is torsion free. Therefore $H$ is an endomorphic image of $H^{\prime} . \operatorname{Ext}\left(A, D \oplus C^{\prime}\right)=0$ for all torsion free groups $A$, and as we have just proved, any homomorphic image of $D \oplus C^{\prime}$ is the direct sum of a cotorsion and a divisible group. It follows that $C$ must be cotorsion.

If $H=D \oplus C$, with $D$ divisible and $C$ cotorsion, then $\operatorname{Ext}(A, H)=0$ for all torsion free groups $A$, and hence $H$ is a direct summand of any group $G$ containing it such that $G / H$ is torsion free. If $H$ is a direct summand of any such $G$, then clearly $H$ is an endomorphic image of any such $G$. Thus our theorem is proved.

The torsion group $T$ is a direct summand of every group containing it as its maximal torsion subgroup if and only if $T=D \oplus B$, with $D$ divisible and $B$ of bounded order. (See [1], pg. 187.) Thus, by our theorem, we see that the torsion group $T$ is an endomorphic image of every group containing it as its maximal torsion subgroup if and only if $T=D \oplus B$, with $D$ divisible and $B$ of bounded order.

The solution of II goes as follows. Suppose a basic subgroup of $T$ is an endomorphic image of every group $G$ in which $T$ is the maximal torsion subgroup. Let $T=D \oplus B$, with $D$ divisible and $B$ reduced. Then a basic subgroup of $T$ must be an endomorphic image of $D \oplus B^{\prime}=$ $D \oplus \operatorname{Ext}(Q / Z, B)$. Therefore a basic subgroup of $T$ must be cotorsion, since it is reduced, and since it is torsion, it is of bounded order. (See [1], pg. 187. The remark by Harrison in [2], pg. 371 is incorrectly worded.) Writing $T$ as $D \oplus B$, we see that a basic subgroup of $B$ is a basic subgroup of $T$. But any two basic subgroups of $T$ are isomorphic, and if $B$ has a basic subgroup of bounded order, then $B$ must be of bounded order. In fact, the only basic subgroup of $B$ is $B$ itself. Thus $T=D \oplus B$, with $D$ divisible and $B$ of bounded order. If $T=$ $D \oplus B$, with $D$ divisible and $B$ of bounded order, then $B$ is a basic subgroup of $T$. Now $D \oplus B$, and hence $B$, is a direct summand of any $G$ in which $T$ is the maximal torsion subgroup. Therefore $B$ is an endomorphic image of any such $G$, and hence any basic subgroup of $T$ 
is such an endomorphic image. Thus we see that the answers to questions I and II are the same.

\section{REFERENCES}

1. L. Fuchs, Abelian Groups, Budapest, 1958.

2. D. K. Harrison, In finite Abelian groups and homological methods, Annals of Math., 69 (1959), 366-391.

New Mexico State University

UNIVERSITY PARK, NEW MEXICO 



\section{PACIFIC JOURNAL OF MATHEMATICS}

\section{EDITORS}

Ralph S. Phillips

Stanford University

Stanford, California

F. H. BrownelL

University of Washington

Seattle 5 , Washington
A. L. Whiteman

University of Southern California Los Angeles 7. California

L. J. PAIGE

University of California

Los Angeles 24, California

ASSOCIATE EDITORS
E. F. BECKENBACH
D. DERRY
H. L. ROYDEN
E. G. STRAUS
T. M. CHERRY
M. OHTSUKA
E. SPANIER
F. WOLF

\section{SUPPORTING INSTITUTIONS}

UNIVERSITY OF BRITISH COLUMBIA

CALIFORNIA INSTITUTE OF TECHNOLOGY

UNIVERSITY OF CALIFORNIA

MONTANA STATE UNIVERSITY

UNIVERSITY OF NEVADA

NEW MEXICO STATE UNIVERSITY

OREGON STATE COLLEGE

UNIVERSITY OF OREGON

OSAKA UNIVERSITY

UNIVERSITY OF SOUTHERN CALIFORNIA
STANFORD UNIVERSITY

UNIVERSITY OF TOKYO

UNIVERSITY OF UTAH

WASHINGTON STATE COLLEGE,

UNIVERSITY OF WASHINGTON

AMERICAN MATHEMATICAL SOCIETY

CALIFORNIA RESEARCH CORPORATION

HUGHES AIRCRAFT COMPANY

SPACE TECHNOLOGY LABORATORIES

NAVAL ORDNANCE TEST STATION

Printed in Japan by International Academic Printing Co., Ltd., Tokyo, Japan

Reprinted 1966 in the United States of America 


\section{Pacific Journal of Mathematics}

\section{Vol. 11, No. $1 \quad$ November, 1961}

A. A. Albert, Generalized twisted fields ............................ 1

Richard Arens, Operational calculus of linear relations ................... 9

John Herbert Barrett, Disconjugacy of a self-adjoint differential equation of the fourth order ....................................... 25

Paul Richard Beesack, Hardy's inequality and its extensions ............... 39

Julius Rubin Blum and David Lee Hanson, On invariant probability measures.

II .............................................

Robert Allen Bonic, Symmetry in group algebras of discrete groups.......... 73

R. Creighton Buck, Multiplication operators ...................... 95

Jack Gary Ceder, Some generalizations of metric spaces ................. 105

Meyer Dwass, Random crossings of cumulative distribution functions ......... 127

Albert Edrei, Wolfgang H. J. Fuchs and Simon Hellerstein, Radial distribution and

deficiencies of the values of a meromorphic function ............... 135

William Cassidy Fox, Harmonic functions with arbitrary local singularities ..... 153

Theodore Thomas Frankel, Manifolds with positive curvature ............... 165

Avner Friedman, A strong maximum principle for weakly subparabolic

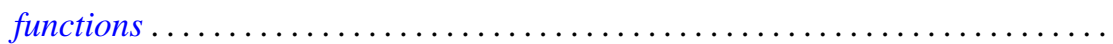

Watson Bryan Fulks and J. O. Sather, Asymptotics. II. Laplace's method for

multiple integrals ......................................

Adriano Mario Garsia and Eugene Richard Rodemich, An embedding of Riemann

surfaces of genus one ..................................... 193

Irving Leonard Glicksberg, Weak compactness and separate continuity......... 205

Branko Grünbaum, On a conjecture of H. Hadwiger .................. 215

Frank J. Hahn, On the action of a locally compact group on $E_{n} \ldots \ldots \ldots \ldots \ldots . . \ldots 221$

Magnus R. Hestenes, Relative hermitian matrices ..................... 225

G. K. Kalisch, On similarity invariants of certain operators in $L_{p} \ldots \ldots \ldots \ldots .247$

Yitzhak Katznelson and Walter Rudin, The Stone-Weierstrass property in Banach

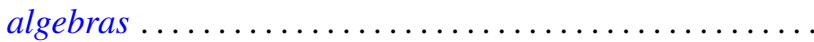

Samir A. Khabbaz, The subgroups of a divisible group $G$ which can be represented as intersections of divisible subgroups of $G \ldots \ldots \ldots \ldots \ldots \ldots \ldots \ldots \ldots . \ldots \ldots 7$

Marvin Isadore Knopp, Construction of a class of modular functions and forms .......................................... 275

Charles Alan McCarthy, Commuting Boolean algebras of projections .......... 295

T. M. MacRobert, Transformations of series of E-functions ................ 309

Heinz Renggli, An inequality for logarithmic capacities ................. 313

M. S. Robertson, Applications of the subordination principle to univalent functions .......................................... 315

David Sachs, Partition and modulated lattices ..................... 325

Frank S. Scalora, Abstract martingale convergence theorems ............... 347

Elbert A. Walker, Torsion endomorphic images of mixed Abelian groups ........ 375

Morgan Ward, The prime divisors of Fibonacci numbers................. 379

Charles R. B. Wright, On the nilpotency class of a group of exponent four....... 387 